\title{
MAPK pathway: a potential target for the treatment of non-small-cell lung carcinoma
}

\author{
Rajesh Pradhan ${ }^{1}$, Gautam Singhvi ${ }^{1}$, Sunil Kumar Dubey ${ }^{\ddagger 1}{ }^{1}$, Gaurav Gupta*, ${ }^{\star 2}$ \& Kamal \\ Dua $^{3,4,5}$ \\ ${ }^{1}$ Department of Pharmacy, Birla Institute of Technology \& Science (BITS), Pilani 333031, India \\ ${ }^{2}$ School of Pharmaceutical Sciences, Jaipur National University, Jagatpura, Jaipur 302017, India \\ ${ }^{3}$ Discipline of Pharmacy, Graduate School of Health, University of Technology Sydney, Ultimo NSW 2007, Australia \\ ${ }^{4}$ School of Biomedical Sciences \& Pharmacy, The University of Newcastle, Callaghan, NSW 2308, Australia \\ ${ }^{5}$ Priority Research Centre for Healthy Lungs, Hunter Medical Research Institute, Lot 1 Kookaburra Circuit, New Lambton Heights, \\ Newcastle, NSW 2305, Australia \\ *Author for correspondence: gauravpharma25@gmail.com \\ $\ddagger$ Authors contributed equally
}

\section{"6focus on molecular level changes of the MAPK pathway and their therapeutic significance in NSCLC"}

First draft submitted: 17 September 2018; Accepted for publication: 28 January 2019; Published online: 17 April 2019

Keywords: apoptosis $\bullet$ cancer $\bullet$ cell proliferation $\bullet$ EGFR $\bullet$ epidermal growth factor receptor $\bullet$ lung cancer $\bullet$ MAPK - MAPK pathway • non-small-cell lung carcinoma • RAS

Lung cancer is a leading cause of cancer-associated death worldwide [1,2]. Explanation of the molecular biology and pathogenesis of lung cancer is essential for the design of a potential treatment for patients [3,4]. Non-small-cell lung carcinoma (NSCLC) is a complex process concerning interruption of cell proliferation, cell differentiation, apoptosis and various molecular mechanisms. The abnormal expression of MAPKs is a reasonably frequent event in NSCLC. The MAPK pathway plays an important role in cell proliferation, differentiation and apoptosis [5]. Increasing evidence supports the association of MAPK signaling deregulation with various types of malignant tumors, including NSCLC. Several studies have demonstrated that members of the MAPK signaling pathway may be potential biomarkers for predicting the progression and prognosis of patients with NSCLC. Additionally, the MAPK pathway affects decisive roles in the carcinogenesis and treatment resistance of NSCLC cells by promoting proliferation or inhibiting apoptosis of NSCLC cells. In this editorial, we will focus on molecular level changes of the MAPK pathway and their therapeutic significance in NSCLC, concentrating primarily on its components which are associated with cell proliferation, cell survival or patient prognosis and potential therapy for NSCLC.

\section{Biological outlines of MAPK pathway}

MAPKs are enzymes belonging to a large family of serine/threonine protein kinases. MAPK plays a major role in directing proliferating activity from the cell cytoplasm to the nucleus and is also involved in regulation of numerous unknown cellular processes (such as differentiation and survival) [5,6]. This signaling process initiated by transmitting signals from external stimuli, for example, hormones, growth factors, cytokines and intracellular essential molecules. Furthermore, the three most important subfamilies of MAPK include the c-Jun N-terminal (JNK; also referred to as stress-activated protein kinases [SAPK]), MAPK14 and the extracellular-signal-regulated kinases (ERK MAPK, Ras/Raf1/MEK/ERK). The ERK MAPK, Ras/Raf1/MEK/ERK are mostly involved in the apoptosis, pathogenesis, progression and oncogenic behavior of lung cell. RAS proto-oncogene specifically plays a crucial role in the transduction of growth-promoting signals and cellular proliferation [6]. Thus, activation of this MAPK signaling pathway is one of the most significant with respect to cell differentiation which subsequently leads to the desired biotic events. 


\section{Molecular mechanistic of MAPK pathway in non-small-cell lung cancer}

In general, the MAPK signaling pathway is involved in various unknown cellular functions, including cell proliferation, differentiation, cell survival, apoptosis and metastasis. Activation and overexpression of this pathway can lead to atypical gene expression and may ultimately result in the malignancy of lung cells. Primarily, there are four major mechanisms involved in the development of non-small-cell lung malignancy [6].

\section{Deregulation of EGFR}

EGFR, a glycoprotein present in the cell surface that regulates the signaling pathway to control cellular proliferation, is stimulated through the binding of a ligand such as EGF or neuregilins [7]. EGFR, also called ErbB-1, is a member of a subfamily of closely related proteins. After ligand binding, EGFR receptor is activated by the intracellular tyrosine kinase domain and initiates autophosphorylation, which causes overexpression of the EGFR and increases the activity of the intracellular pathway. As a result, atypical cellular activity (such as cell proliferation, angiogenesis, invasion and metastasis) occur in non-small lung cells. Deregulation of EGFR has been seen in between 40 and $89 \%$ of NSCLC $[7]$.

\section{Mutation of the RAS gene}

The RAS gene comprises three subfamilies; H-RAS, K-RAS and N-RAS. These encode for membrane-bound 21-kD guanosine-triphosphate-(GTP-) binding proteins, which regulate various cell responses such as apoptosis and metastasis by means of binding with respective effectors (e.g., MAPK, PI3K and STAT). This may ultimately lead to a mutation in RAS with abnormal GTPase activity, leading to the development of NSCLC. About 20-30\% of adenocarcinoma-related NSCLC results from a mutation in the Ras gene [8].

\section{Mutation of BRAF}

BRAF is a serine/threonine protein kinase. Mutations in BRAF occur through EGFR mutations or ALK rearrangements and have been identified in $2-4 \%$ of NSCLC.

Additionally, activation of the downstream transcription factor c-Jun and auto-phosphorylation of MAPK pathway possibly plays a crucial role in differentiation, apoptosis and metastasis. Typically, increased c-Jun Nterminal kinase activity has been seen in human cancers $[6,8]$.

\section{Regulation of MAPK signaling pathway: a novel trend of management for NSCLC}

In this recent year, the MAPK pathway (Ras, Raf and MEK) has been found to be a viable therapeutic target for novel treatments for cancer. Relevant drugs should be specific for their targets and conceivably less toxic than traditional cancer therapy $[9,10]$. EGFR inhibitors actively bind to EGFR, subsequently blocking the binding of alternative ligands, preventing the overexpression and proliferative activity of EGFR in NSCLC. Gefitinib and erlotinib are EGFR inhibitors which are both already available on the market and may possibly be used as a treatment for NSCLC [11-14].

Subsequently, MAPK (MEK) inhibitors are downstream effectors which inhibit NSCLC by blocking the activation of the MAPK signaling cascade. For instance, PD-184352 (an orally active difluorobenzamide) is an MEK inhibitor which has displayed inhibitory action in preclinical studies and Phase I clinical trials [11,15]. The Phase I clinical study concluded that the drug was well tolerated and safe; however, the Phase II clinical study observed lack of efficacy, resulting in the withdrawal of this drug [11]. Hence, another second-generation MEK inhibitor (PD0325901) has entered clinical trials; and thankfully, it has displayed better therapeutic activity and pharmaceutical properties than PD184352 [11,16]. Based on this and because of the documented activation of the MEK pathway in NSCLC, MEK is still considered as an effective objective for NSCLC.

Moreover, LGX818 is a BRAF inhibitor, which inhibits the proliferation of the malignancy cell line by blocking multi-signaling pathways such as ERK phosphorylation, RAF kinase inhibitors and mTOR inhibitors. This may have great medicinal activity and lead to a novel trend for the treatment of NSCLC $[17,18]$.

\section{Conclusion}

Over the past two decades, noteworthy progress has been emphasized in NSCLC treatment. A better understanding of the cancer biology has allowed the development of novel targeted approaches for the treatment of carcinoma. Moreover, MAPK signaling pathways are a crucial factor in NSCLC and have played a major role in the improvement of treatment of this carcinoma. This prospect has created an opportunity for the development of novel compounds 
for the treatment of NSCLC. These moieties are not only important for cell survival and proliferation but can also promote the malignant cell death, apoptosis and metastasis. However, there are numerous complications to the ideal exploration of targeted novel therapeutics. To improve this approach, unending efforts are needed to find novel molecular mechanisms and compounds.

\section{Financial \& competing interests disclosure}

The authors have no relevant affiliations or financial involvement with any organization or entity with a financial interest in or financial conflict with the subject matter or materials discussed in the manuscript. This includes employment, consultancies, honoraria, stock ownership or options, expert testimony, grants or patents received or pending, or royalties.

No writing assistance was utilized in the production of this manuscript.

\section{References}

1. El-Telbany A, Ma PC. Cancer genes in lung cancer: racial disparities: are there any? Genes Cancer 3(7-8), 467-480 (2012).

2. Woods A, Rahman KM. Antimicrobial molecules in the lung: formulation challenges and future directions for innovation. Future Med. Chem. 10(5), 575-604 (2018).

3. Zou B, Zhou X-L, Lai S-Q, Liu J-C. Notch signaling and non-small-cell lung cancer. Oncol. Lett. 15(3), 3415-3421 (2018).

4. Pifano M, Garona J, Sobol NT, Alberto M, Alonso DF, Ripoll GV. Search of vasopressin analogs with antiproliferative activity on small-cell lung cancer: drug design based on two different approaches. Future Med. Chem. 10(8), 879-894 (2018).

5. Peng Q, Deng Z, Pan H, Gu L, Liu O, Tang Z. Mitogen-activated protein kinase signaling pathway in oral cancer. Oncol. Lett. 15(2), 1379-1388 (2018).

6. Fang JY, Richardson BC. The MAPK signalling pathways and colorectal cancer. Lancet Oncol. 6(5), 322-327 (2005).

7. Bethune G, Bethune D, Ridgway N, Xu Z. Epidermal growth factor receptor (EGFR) in lung cancer: an overview and update. J. Thorac. Dis. 2(1), 48-51 (2010).

8. Abdel-Rahman O. Targeting the MEK signaling pathway in non-small-cell lung cancer (NSCLC) patients with RAS aberrations. Ther. Adv. Respir. Dis. 10(3), 265-274 (2016).

9. Santarpia L, Lippman SM, El-Naggar AK. Targeting the MAPK-RAS-RAF signaling pathway in cancer therapy. Expert Opin. Ther. Targets 16(1), 103-119 (2012).

10. Hilger RA, Scheulen ME, Strumberg D. The Ras-Raf-MEK-ERK pathway in the treatment of cancer. Onkologie 25(6), 511-518 (2002).

11. Cheng Y, Tian H. Current development status of MEK inhibitors. Molecules 22(10), 1-20 (2017).

12. Wang Y, Schmid-Bindert G, Zhou C. Erlotinib in the treatment of advanced non-small-cell lung cancer: an update for clinicians. Ther. Adv. Med. Oncol. 4(1), 19-29 (2012).

13. Ganjoo KN, Wakelee H. Review of erlotinib in the treatment of advanced non-small-cell lung cancer. Biologics 1(4), 335-346 (2007).

14. Nurwidya F, Takahashi F, Takahashi K. Gefitinib in the treatment of nonsmall cell lung cancer with activating epidermal growth factor receptor mutation. J. Nat. Sci. Biol. Med. 7(2), 119-123 (2016).

15. Sebolt-Leopold J, Dudley DT, Herrera R et al. Blockade of the MAP kinase pathway suppresses growth of colon tumors in vivo. Nat. Med. 5, 810-816 (1999).

16. Henderson YC, Chen Y, Frederick MJ, Lai SY, Clayman GL. MEK inhibitor PD0325901 significantly reduces the growth of papillary thyroid carcinoma cells in vitro and in vivo. Mol. Cancer Ther. 9(7), 1968-1976 (2010).

17. Baik CS, Myall NJ, Wakelee HA. Targeting BRAF-mutant non-small-cell lung cancer: from molecular profiling to rationally designed therapy. Oncologist 22(7), 786-796 (2017).

18. Griffin M, Scotto D, Josephs DH et al. BRAF inhibitors: resistance and the promise of combination treatments for melanoma. Oncotarget 8(44), 78174-78192 (2017). 
